\title{
Vitrectomia transconjuntival: dados preliminares com o sistema Millennium TSV-25
}

\author{
Transconjunctival vitrectomy:preliminarydata using the TSV-25 Millennium System
}

\author{
Flávio Rezende Filho ${ }^{1}$ \\ Simone Alcântara ${ }^{2}$ \\ Luiz Gustavo Tonelli Régis ${ }^{3}$
}

\section{RESUMO}

Objetivo: Relatar os resultados cirúrgicos preliminares de vitrectomia via pars plana com técnica transconjuntival com o sistema Millennium-TSV25. Métodos: Vinte pacientes (20 olhos), obtidos seqüencialmente, submetidos à vitrectomia transconjuntival com o sistema MillenniumTSV-25, entre julho de 2003 e janeiro de 2004 , foram avaliados prospectivamente por um período mínimo de um mês pós-operatório. Olhos com buraco macular, membrana epi-retiniana, edema macular, descolamento regmatogênico da retina, endoftalmite e hemorragia vítrea com ou sem descolamento tracional da retina foram incluídos. Complicações e dificuldades intra e pós-operatórias foram computadas. Resultados: Os 20 pacientes completaram pelo menos um mês de acompanhamento pósoperatório. Foram 4 buracos maculares, 2 membranas epi-retinianas, 2 edemas maculares cistóides pós-facectomia, 1 edema macular diabético, 5 descolamentos regmatogênicos da retina, 5 hemorragias vítreas (2 associadas a descolamento tracional da retina) e 1 endoftalmite. A média da pressão intra-ocular no primeiro dia pós-operatório foi de $15,7 \mathrm{mmHg}$ (6- $46 \mathrm{mmHg})$ e no primeiro mês de $14,2 \mathrm{mmHg}(8-22 \mathrm{mmHg})$. Não houve nenhum caso de complicação, como rotura relacionada às esclerotomias, endoftalmite ou hipotonia ocular pós-operatória. Três dos 5 olhos (60\%) com descolamento de retina necessitaram de nova intervenção cirúrgica (todos pseudofácicos) e 4 dos 5 olhos (80\%) com hemorragia vítrea tiveram hemorragia vítrea residual pós-operatória. Todos os casos com alterações maculares e os olhos fácicos com descolamento de retina foram bem sucedidos. Conclusões: Esta técnica de vitrectomia transconjuntival demonstrou, como principais benefícios, a baixa incidência de roturas relacionadas às esclerotomias e hipertensão ocular pós-operatória. Parece ser imprescindível a adequada seleção dos casos.

Descritores: Corpo vítreo/cirurgia; Vitrectomia/métodos; Complicações pós-operatórias; Microscopia/métodos; Ultra-sonografia/métodos; Procedimentos cirúrgicos minimamente invasivos; Resultado de tratamento

\section{INTRODUÇÃO}

Desde a introdução da chamada moderna vitrectomia, realizada a céu aberto por Kasner, em 1969, um dos grandes avanços nas técnicas cirúrgicas vítreo-retinianas tem sido a diminuição do tamanho da incisão ${ }^{(1)}$. Três anos após Kasner, Machemer et al., já apresentavam resultado de 100 casos operados por vitrectomia via pars plana (VVPP) em sistema fechado, utilizando o "vitreous-infusion-suction-cutter" (VISC) ${ }^{(2)}$. O VISC necessitava de esclerotomia de $3,5 \mathrm{~mm}$, pois, tanto o corte e a aspiração, quanto à irrigação e a iluminação, eram executadas pelo mesmo instrumento. O índice 
de roturas relacionadas às esclerotomias relatado por estes autores foi de $20 \%{ }^{(2)}$.

Também em 1972, O'Malley e Heintz já separaram as funções de iluminação e irrigação do instrumento de corte $^{(3)}$. Este avanço, dentre inúmeras outras vantagens, possibilitou a diminuição das esclerotomias. Em 1977, Tardif et al., reportaram suas complicações relacionadas às esclerotomias, incluindo laceração do corpo ciliar, encarceramento vítreo, crescimento fibrovascular através das esclerotomias (fibrovascular ingrowth) e $12 \%$ de roturas associadas às esclerotomias ${ }^{(4)}$.

Atualmente, a VVPP é realizada convencionalmente através de 3 incisões esclerais distintas com, aproximadamente, $1 \mathrm{~mm}$ de diâmetro cada, feitas após a conjuntivotomia. Estas esclerotomias podem, ainda, ser auto-selantes, através de túneis esclerais ${ }^{(5)}$.

Singh et al. em 1996, introduziram um sistema para biópsia vítrea nos casos de endoftalmite, onde realizavam vitrectomia transconjuntival ${ }^{(6)}$. Porém, este equipamento não permitia a troca de instrumentos, servindo, apenas, para rápidas intervenções ${ }^{(7)}$.

Recentemente, Fujii et al., desenvolveram um novo sistema de vitrectomia transconjuntival através de 3 incisões de $0,5 \mathrm{~mm}$, que permite a troca de instrumentos durante a cirurgia. Os autores alegam ser esta uma técnica menos invasiva, sem perder a eficácia durante o procedimento ${ }^{(8)}$.

O objetivo do presente estudo é reportar as complicações intra e pós-operatórias precoces, dificuldades e possíveis benefícios desta técnica em pacientes brasileiros.

\section{MÉTODOS}

Vinte pacientes (20 olhos) obtidos seqüencialmente, submetidos à vitrectomia transconjuntival, foram analisados prospectivamente. Dados intra e pós-operatórios foram registrados. O acompanhamento mínimo pós-operatório foi de 1 mês, sendo que os pacientes submetidos a tamponamento com gás (hexafluoreto de enxofre - $\mathrm{SF}_{6}$ ) foram acompanhados por pelo menos 2 semanas após a absorção completa do gás. $\mathrm{O}$ estudo foi conduzido, na íntegra, no Centro de Estudos e Pesquisas Oculistas Associados - Rio de Janeiro (CEPOA).

A tabela 1 lista as indicações cirúrgicas dos olhos incluídos no estudo. Pacientes com diagnóstico pré-operatório de descolamento regmatogênico da retina (DRR) com vitreorretinopatia proliferativa avançada, em que tamponamento com óleo de silicone era programado, olhos com descolamentos tracionais da retina extensos e olhos com glaucoma não foram incluídos. Os pacientes pseudofácicos com DRR apresentavam história prévia de rotura de cápsula posterior durante a facectomia.

Todos os olhos com hemorragia vítrea ou DRR foram submetidos à ultra-sonografia $\mathrm{B}$ (USB) (B-Scan S, Biovision, França) para detecção do tipo de descolamento posterior do vítreo e para excluir outras doenças concomitantes que poderiam contra-indicar esta técnica. Os olhos com doenças maculares foram submetidos à tomografia de coerência óptica (TCO) (Stratus OCT - modelo 3000, Carl Zeiss Meditec, Alemanha) pré e pós-operatória.

Dados pré-operatórios registrados incluíram gênero, ida- de, estado do cristalino (fácico ou pseudofácico), pressão intra-ocular (PIO) e indicação cirúrgica. O exame clínico pré e pós-operatório envolveu biomicroscopia do segmento anterior e da retina, tonometria de aplanação e oftalmoscopia binocular indireta com depressão escleral.

As visitas pós-operatórias foram feitas no primeiro dia, primeira semana e primeiro mês, e os principais dados registrados foram PIO, presença ou ausência de roturas associadas às esclerotomias, presença de descolamento de coróide ou qualquer outra alteração importante de segmento anterior ou posterior.

Todas as cirurgias foram realizadas pelo mesmo cirurgião vítreo-retiniano com o sistema Millennium TSV-25 (Bausch \& Lomb Surgical, Brasil) e utilização de lentes de grande angular de contato ou de não-contato. A técnica cirúrgica utilizada foi a descrita, originalmente, por Fujii et al.$^{(8)}$, e, recentemente, detalhada pelo autor ${ }^{(9)}$. Após as incisões via transconjuntival $(0,5 \mathrm{~mm}$ cada), retira-se a lança de esclerotomia e mantém-se um trocater por onde os instrumentos entram e saem do olho. A linha de infusão é autofixada. A pressão de infusão é variável durante o ato cirúrgico, mas, inicialmente, é de $44 \mathrm{mmHg}$. Durante a cirurgia, utilizou-se a freqüência de corte fixa em 1.500 cortes por minuto e aspiração de $550 \mathrm{mmHg}$. Ao término da cirurgia, os trocateres foram retirados sem a necessidade de suturas.

No paciente 11 da tabela 1 , foi necessário a utilização de substância viscoelástica (metilcelulose, Ophthalmos, São Paulo) para uma melhor visualização do segmento posterior através da câmara anterior do olho operado.

Foi obtido, pré-operatoriamente, o consentimento informado de todos os pacientes que ingressaram no estudo, de acordo com as orientações da aprovação do comitê de ética do CEPOA.

\section{RESULTADOS}

Todos os vinte pacientes recrutados concluíram, pelo menos, 1 mês de acompanhamento pós-operatório. As complicações pós-operatórias e a variação da PIO estão descritas na tabela 2 . A PIO média pré-operatória foi de $14,1 \mathrm{mmHg}$ (variando de 7 a $25 \mathrm{mmHg}$ ), enquanto que, no primeiro dia pós-operatório, foi de $15,7 \mathrm{mmHg}$ (6 - $46 \mathrm{mmHg}$ ), na primeira semana, de $15,1 \mathrm{mmHg}$ (10-27 mmHg)e, no primeiro mês, foi de $14,2 \mathrm{mmHg}(8-22 \mathrm{mmHg})$. Os quatro olhos com buraco macular idiopático obtiveram fechamento completo. Todos os demais pacientes com alterações maculares ( 2 membranas epirretinianas, 2 edemas maculares cistóides pós-facectomia e 1 edema macular diabético) (Tabela 1) também obtiveram sucesso cirúrgico anatômico, com diminuição da espessura macular documentada por TCO ao final de 1 mês de acompanhamento (Figura 1). A ausência de sutura nas esclerotomias e conjuntiva promoveu uma cicatrização pós-operatória com pouca reação inflamatória local.

\section{Complicações intra-operatórias}

Em 2 pacientes, um trocater saiu durante a troca de instrumentos e estes tiveram que ser reintroduzidos. Em três olhos, houve vazamento de gás subconjuntival antes da remoção da linha de infusão. Todos os olhos mantiveram-se normotensos 


\begin{tabular}{|c|c|c|c|c|c|}
\hline Paciente & Olho & Sexo & Idade & Cristalino & Diagnóstico \\
\hline 1 & OD & $\mathrm{F}$ & 53 & Fácico & Buraco macular \\
\hline 2 & OD & $\mathrm{F}$ & 88 & Pseudo-fácico & Hemorragia vítrea \\
\hline 3 & OD & M & 62 & Fácico & Descolamento regmatogênico da retina \\
\hline 4 & $\mathrm{OE}$ & $\mathrm{F}$ & 51 & Fácico & Descolamento regmatogênico da retina \\
\hline 5 & OE & M & 56 & Fácico & Hemorragia vítrea e descolamento tracional da retina \\
\hline 6 & OE & $\mathrm{F}$ & 58 & Fácico & Buraco macular \\
\hline 7 & $\mathrm{OE}$ & M & 74 & Fácico & Hemorragia vítrea \\
\hline 8 & OD & M & 60 & Fácico & Buraco macular \\
\hline 9 & OD & $\mathrm{F}$ & 89 & Pseudo-fácico & Endoftalmite pós-facectomia \\
\hline 10 & OD & M & 72 & Pseudo-fácico & Descolamento regmatogênico da retina \\
\hline 11 & OD & M & 68 & Pseudo-fácico & Descolamento regmatogênico da retina \\
\hline 12 & OE & $\mathrm{F}$ & 66 & Pseudo-fácico & Membrana epi-retiniana \\
\hline 13 & OE & $\mathrm{F}$ & 85 & Pseudo-fácico & Edema macular cistóide e restos corticais pós-facectomia \\
\hline 14 & OE & $\mathrm{F}$ & 68 & Fácico & Buraco macular \\
\hline 15 & $\mathrm{OE}$ & M & 75 & Pseudo-fácico & Edema macular cistóide pós-facectomia \\
\hline 16 & OE & $\mathrm{F}$ & 69 & Pseudo-fácico & Edema macular diabético \\
\hline 17 & OE & $\mathrm{F}$ & 71 & Fácico & Membrana epi-retiniana \\
\hline 18 & OD & M & 64 & Fácico & Hemorragia vítrea \\
\hline 19 & OD & M & 70 & Pseudo-fácico & Hemorragia vítrea e descolamento tracional da retina \\
\hline 20 & OE & $\mathrm{F}$ & 68 & Pseudo-fácico & Descolamento regmatogênico da retina \\
\hline
\end{tabular}

\begin{tabular}{|c|c|c|c|c|c|}
\hline Paciente & PIO pré-op & $\begin{array}{l}\text { PIO pós-op } \\
\text { (10 dia) }\end{array}$ & $\begin{array}{l}\text { PIO pós-op } \\
\text { (19 semana) }\end{array}$ & $\begin{array}{l}\text { PIO pós-op } \\
\text { (10 mês) }\end{array}$ & Complicações pós-op \\
\hline 1 & 13 & 10 & 20 & 16 & - \\
\hline 2 & 9 & 6 & 10 & 12 & - \\
\hline 3 & 17 & 15 & 16 & 18 & - \\
\hline 4 & 13 & 15 & 12 & 15 & - \\
\hline 5 & 13 & 23 & 17 & 10 & DRR com rotura no pólo posterior e hemorragia vítrea residual \\
\hline 6 & 16 & 17 & 15 & 11 & - \\
\hline 7 & 12 & 15 & 22 & 18 & Hemorragia vítrea residual \\
\hline 8 & 14 & 12 & 15 & 16 & - \\
\hline 9 & 10 & 15 & 15 & 10 & - \\
\hline 10 & 7 & 21 & 18 & 20 & DRR devido à reabertura da rotura \\
\hline 11 & 12 & 46 & 27 & 14 & DRR devido à reabertura da rotura \\
\hline 12 & 16 & 12 & 12 & 10 & - \\
\hline 13 & 12 & 9 & 10 & 12 & - \\
\hline 14 & 16 & 12 & 12 & 10 & - \\
\hline 15 & 16 & 11 & 10 & 18 & - \\
\hline 16 & 19 & 20 & 16 & 16 & - \\
\hline 17 & 14 & 18 & 15 & 14 & - \\
\hline 18 & 25 & 15 & 13 & 14 & Hemorragia vítrea residual \\
\hline 19 & 14 & 10 & 17 & 22 & Hemorragia vítrea residual \\
\hline 20 & 14 & 12 & 10 & 8 & DRR devido à reabertura da rotura \\
\hline
\end{tabular}

após a remoção dos trocateres e da linha de infusão. Não houve necessidade de sutura das esclerotomias por vazamento em nenhum paciente. Apesar do pequeno calibre da linha de infusão (infusão mantida entre 29 e $44 \mathrm{mmHg}$ ), nenhum olho teve hipotonia ocular intra-operatória (clinicamente traduzida como dobras na coróide).

\section{Complicações pós-operatórias}

Dos cinco olhos com hemorragia vítrea pós-operatória, quatro apresentaram hemorragia vítrea residual no pós-operatório, sendo que dois destes olhos apresentavam associados descolamento tracional da retina no pré-operatório.

Todos os olhos com DRR apresentavam descolamento 
posterior do vítreo parcial no pré-operatório, documentado por USB, e rotura retiniana por tração vítrea. Os dois pacientes fácicos, com roturas mais posteriores, obtiveram sucesso cirúrgico (em ambos, as roturas estavam localizadas no limite posterior do equador, às 3:00 horas). Os 3 pacientes pseudofácicos, com roturas mais periféricas (margem posterior da base vítrea), necessitaram de re-operação devido à reabertura das roturas primárias, sendo que um deles, com rotura inferior (às 6:00 horas), desenvolveu contração da superfície retiniana no local da rotura. Os outros 2 apresentavam roturas superiores, próximas às esclerotomias (entre 11:00 e 1:00 hora). O paciente 11 desenvolveu aumento significativo da PIO no primeiro dia pós-operatório e foi o único que precisou de medicação hipotensora ocular durante o estudo (Tabela 2).

Não houve nenhum caso de rotura retiniana relacionada às esclerotomias, de endoftalmite, de fibrina na câmara anterior e de hipotonia ocular pós-operatória.

\section{COMENTÁRIOS}

Analisando estes dados preliminares, os autores identificaram pontos positivos e alguns potencialmente negativos relacionados a esta nova técnica de vitrectomia transconjuntival.

Dos olhos incluídos nesta série, os que tinham doenças maculares (buraco macular, membrana epirretiniana, edema macular cistóide pós-facectomia e edema macular diabético) e os fácicos com DRR foram os que obtiveram os melhores resultados anatômicos preliminares (Figura 1).

A ausência de roturas relacionadas às esclerotomias (nenhum olho em 20) foi o ponto considerado como dos mais importantes. Vale salientar que múltiplas indicações cirúrgicas foram incluídas neste estudo, e diferentes instrumentais cirúrgicos intra-oculares foram utilizados, como endocautério, endolaser, manipuladores de tecidos, pinças e tesouras. Recentemente, Haritoglou et al., obtiveram um índice de 20\% (4 olhos em 20) de DRR, com roturas relacionadas a esclerotomias, após vitrectomia com a técnica convencional para retirada de membrana epirretiniana utilizando indocianina verde ${ }^{(10)}$. O diâmetro das esclerotomias com esta nova técnica é reduzido pela metade.

Gonzales et al., utilizando biomicroscopia ultra-sônica (UBM) para comparar as esclerotomias convencionais (1,0 mm) com as desse novo sistema $(0,5 \mathrm{~mm})$, não encontraram diferença significativa no tipo e tempo de cicatrização e nem na quantidade de encarceramento vítreo ${ }^{(11)}$. Porém, a figura 2 ilustra a diferença de encarceramento vítreo comparando os dois tipos de incisão em olhos com hemorragia vítrea e retinopatia diabética proliferativa. Estudos futuros poderão esclarecer se esta diferença tem influência no índice de crescimento fibrovascular através das esclerotomias.

Outro fator que também pode contribuir para a aparente diminuição na incidência de roturas relacionadas às esclerotomias é a utilização de trocateres. Territo et al., demonstraram que o uso de trocateres em esclerotomias convencionais diminui a incidência das roturas a elas relacionadas e especularam que tal fato possa ser oriundo da proteção à base vítrea que estes trocateres promovem durante a troca de instrumen$\operatorname{tos}^{(12)}$. A baixa incidência dessas roturas com o sistema de vitrectomia transconjuntival também foi encontrada por Yepremyan e Kapusta ${ }^{(13)}$.

Uma vez que o encarceramento vítreo é o que parece ser necessário para manter a incisão auto-selante nos primeiros dias após a cirurgia, no momento, é recomendado que se deixe mais vítreo na periferia ${ }^{(14)}$. Este fator pode ser um problema

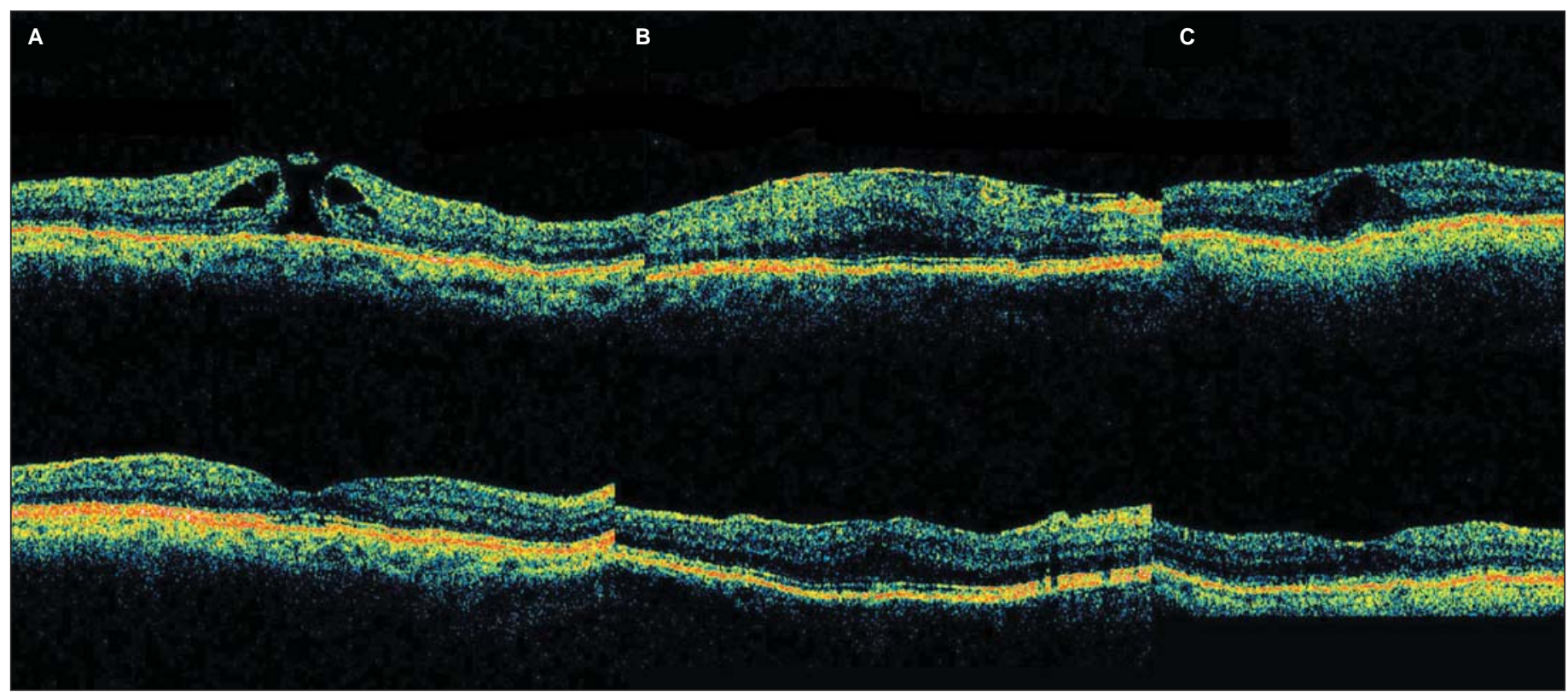

Figura 1 - Tomografia de coerência óptica pré- (acima) e pós-operatória (abaixo) ilustrando alguns dos olhos com alterações maculares operados com a técnica de vitrectomia transconjuntival. A: Buraco macular idiopático; B: Membrana epi-retiniana; C: Edema macular cistóide pós-facectomia 


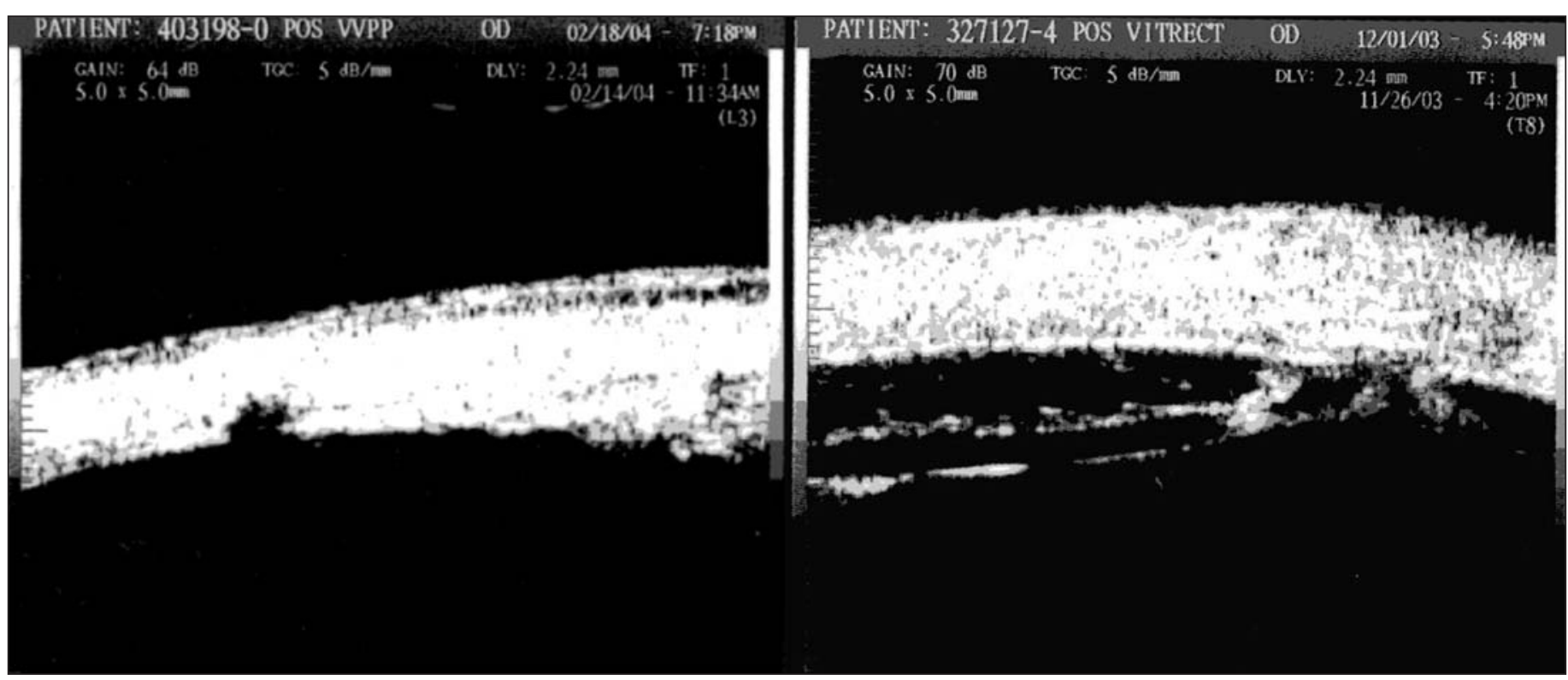

Figura 2 - Ultra-sonografia biomicroscópica (UBM) das esclerotomias: À esquerda, olho de um dos pacientes do estudo (incisão de 0,5 mm) Nota-se discreta descontinuidade apenas da pars plana e parte mais interna da esclera, sem a presença de encarceramento vítreo evidente. À direita, olho feito com técnica convencional (incisão de $1,0 \mathrm{~mm}$ ). Nota-se evidente encarceramento vítreo na incisão

para olhos pseudo-fácicos com descolamento de retina e roturas periféricas. A presença deste vítreo residual poderá exercer tração suficiente que mantenha estas roturas abertas e diminua o índice de sucesso nestes casos, podendo, até mesmo, facilitar o aparecimento de tração vítreo-retiniana. Isto parece ter sido demonstrado no presente estudo, em que os 3 olhos pseudo-fácicos com DRR e roturas periféricas redescolaram a retina no pós-operatório devido à tração mantida nas roturas primárias. Por outro lado, os 2 olhos fácicos com roturas mais posteriores foram bem sucedidos com esta técnica. Porém, é importante frisar que todos os olhos pseudofácicos apresentavam rotura na cápsula posterior, com precária visualização da periferia retiniana, e, em 2 dos 3 olhos pseudo-fácicos, as roturas estavam próximas às esclerotomias superiores, tornando mais difícil o acesso a elas.

O encarceramento vítreo na incisão também pode facilitar a entrada de microorganismos na cavidade vítrea e aumentar o índice de endoftalmite pós-operatória ${ }^{(14)}$. No entanto, o desalinhamento da incisão conjuntival com a esclerotomia parece dificultar este acesso.

Neste estudo, apenas 1 paciente $(5 \%)$ necessitou de medicação hipotensora ocular (único acima de $30 \mathrm{mmHg}$ ). Esta técnica de vitrectomia transconjuntival parece promover valores de PIO pós-operatória sensivelmente menores que a técnica convencional. Desai et al., relataram uma incidência de 40\% (10 olhos em 25) de PIO acima de $30 \mathrm{mmHg}$ no primeiro dia pós-operatório de VVPP com a técnica convencional para casos simples ${ }^{(15)}$.

O presente estudo não analisou o tempo de recuperação visual e a quantidade de reação inflamatória local, pois o caráter não comparativo e a diversidade dos casos impediriam uma avaliação significativa. Isto deverá ser avaliado em estudos subseqüentes. Porém, Okamoto et al., já demonstraram que as incisões transconjuntivais (25 gauge) sem suturas induzem menos alterações na topografia corneana do que as esclerotomias convencionais suturadas (20 gauge $)^{(16)}$. E Fujii et al., observaram clinicamente que a ausência de suturas nas esclerotomias e conjuntiva provocou menor reação inflamatória local e menor desconforto pós-operatório aos pacientes ${ }^{(17)}$.

Nos casos de hemorragia vítrea, o fato de deixar mais vítreo na periferia também pode contribuir para um maior índice de hemorragia vítrea residual pós-operatória. Isto aconteceu em $80 \%$ (4 dos 5 olhos) dos casos e é consideravelmente maior do que o índice de aproximadamente $40 \%$ relatado por Virata e Kylstra com a técnica convencional (ambos os estudos utilizando sistema de visualização de grande angular) ${ }^{(18)}$.

O menor calibre das esclerotomias requer instrumentos mais delicados, como vitreófago, sondas de iluminação e endolaser, pinças, tesouras e cautério intra-ocular. Os materiais atualmente disponíveis são maleáveis e, com freqüência, tornam-se curvos durante a cirurgia. Isto dificulta a manipulação do olho durante os procedimentos ${ }^{(14)}$. Os instrumentos que se encurvaram foram a sonda de iluminação e o cautério intra-ocular. Durante a cirurgia do paciente 5 (Tabela 1), o cautério intra-ocular quebrou em sua base, sem causar danos ao olho. Isto é uma complicação potencialmente grave e ilustra a necessidade de aprimora mento da rigidez e durabilidade destes instrumentais.

O sistema de trocater também necessita ser aprimorado, sendo que, em 2 casos (pacientes 11 e 18 - Tabela 1), um dos trocateres saiu durante a cirurgia. A dificuldade na introdução dos trocateres varia de acordo com a rigidez escleral de cada paciente e diminui com a maior experiência do cirurgião.

O calibre reduzido da linha de infusão diminui em quase 7 vezes a velocidade de entrada da solução salina balanceada na cavidade vítrea ${ }^{(8)}$, sendo necessário cuidado intra-operatório 
para que não haja hipotonia. Nesta série, não houve nenhum caso de descolamento de coróide intra- ou pós-operatório.

Outro fator relacionado à diminuição do calibre dos instrumentos é que a abertura na ponta do vitreótomo é significativamente menor que a dos vitreótomos convencionais, diminuindo o "flow rate" em, aproximadamente, 2 vezes ${ }^{(8)}$. Porém, os autores não notaram um aumento no tempo cirúrgico intra-ocular. Houve, sim, uma diminuição na quantidade de solução salina balanceada utilizada por caso (média de $100 \mathrm{ml}$, dados não incluídos neste trabalho), pois, com o reduzido calibre das esclerotomias, quase não há vazamento de líquido em volta dos instrumentos.

\section{CONCLUSÃO}

O presente estudo relata os primeiros resultados com a técnica de vitrectomia transconjuntival em nosso meio. A seleção adequada dos casos parece ser fundamental para o sucesso cirúrgico. Os principais pontos positivos deste sistema foram a baixa incidência de roturas relacionadas às esclerotomias e de hipertensão ocular no pós-operatório. Os pontos negativos incluíram a fragilidade dos instrumentos e o insucesso em olhos pseudo-fácicos com DRR. Estudos multicêntricos randomizados deverão esclarecer melhor o papel desta nova técnica de vitrectomia via pars plana no tratamento das doenças vítreo-retinianas.

\section{ABSTRACT}

Purpose: To report the preliminary surgical results of pars plana vitrectomy with a transconjunctival technique using the TSV-25 Millennium system. Methods: Twenty consecutive patients (20 eyes) that underwent transconjunctival vitrectomy with the TSV-25 Millennium system, between July 2003 and January 2004, were prospectively postoperatively followed for a minimum period of one month. Eyes with macular hole, epiretinal membrane, macular edema, rhegmatogenous retinal detachment, endophthalmitis, and vitreous hemorrhage with or without tractional retinal detachment were included. Intra and post-operative complications and difficulties were recorded. Results: All 20 patients completed at least one month postoperative follow-up. There were 4 macular holes, 2 epiretinal membranes, 2 cystoid macular edemas post-cataract surgery, 1 diabetic macular edema, 5 rhegmatogenous retinal detachments, 5 vitreous hemorrhages ( 2 associated with tractional retinal detachment), and 1 endophthalmitis. Mean postoperative day one intraocular pressure was $15.7 \mathrm{mmHg}(6-46 \mathrm{mmHg})$ and at one postoperative month it was $14.2 \mathrm{mmHg}(8-22 \mathrm{mmHg})$. There were no cases of sclerotomy-related retinal breaks, postoperative endophthalmitis, or ocular hypotony. Three of 5 eyes (60\%) with retinal detachment needed further surgical intervention (all pseudophakic) and 4 of 5 eyes (80\%) with vitreous hemorrhage had postoperative residual hemorrhage. All cases with macular diseases and phakic retinal detachments were successful with one surgery. Conclusion: This transconjunctival vitrectomy technique showed as its main benefits the low incidence of sclerotomy-related breaks and postoperative ocular hypertension. An adequate case selection seems to be crucial.

Keywords: Vitreous body/surgery; Vitrectomy/methods; Postoperative complications; Microscopy/instrumentation; Ultrasonography/instrumentation; Surgical procedure minimally invasive; Treatment outcome

\section{REFERÊNCIAS}

1. Kasner D. Vitrectomy: a new approach to management of vitreous. Highlights Ophthalmol. 1969;11:304.

2. Machemer R, Parel JM, Norton EW. Vitrectomy: a pars plana approach. Technical improvements and further results. Trans Am Acad Ophthalmol Otolaryngol. 1972;76(2):462-6.

3. O'Malley C, Heintz RM. Vitrectomy via pars plana: a new instrument system. Trans Pac Coast Otoophthalmol Soc Annu Meet. 1972;53:121-37.

4. Tardif YM, Schepens CL, Tolentino FI. Vitreous surgery. XIV. Complications from sclerotomy in 89 consecutive cases. Arch Ophthalmol. 1977;95(2):229-34.

5. Chen JC. Sutureless pars plana vitrectomy through self-sealing sclerotomies. Arch Ophthalmol. 1996;114(10):1273-5.

6. Singh S, Josephberg RJ, Zaidman GW. Office-based diagnostic pars plana vitrectomy. Invest Ophthalmol Vis Sci. 1996;37:402.

7. Josephberg RG. Sutureless vitrectomy surgery. Ophthalmology. 2003;110 (12):2427; author reply 2427. Erratum in: Ophthalmology. 2004;11(2):331.

8. Fujii GY, de Juan Jr E, Humayun MS, Pieramici DJ, Chang TS, Awh C, et al. A new 25-gauge instrument system for transconjunctival sutureless vitrectomy surgery. Ophthalmology. 2002;109(10):1807-13; discussion 1813. Erratum in: Ophthalmology. 2003;110(1):9.

9. Rezende Filho F. Cirurgiões de vítreo também usam incisão auto-selante. Oftalmol Foco. 2003;86(ago/set):28-9.

10. Haritoglou C, Gandofer A, Gass CA, Schaumberger M, Ulbig MW, Kampik A. The effect of indocyanine-green on functional outcome of macular pucker surgery. Am J Ophthalmol. 2003;135(3):328-37.

11. Gonzales CR, Gupta A, Lee SY, Freeman JY, Estafanous MF, Kreiger AE, et al. Ultrasound biomicroscopic evaluation of sclerotomies created by transconjunctival 25 Gauge vitrectomy. Invest Ophthalmol Vis Sci. 2003;44: E-Abstract 3008.

12. Territo C, Gieser JP, Wilson CA, Anand R. Influence of the cannulated vitrectomy system on the occurence of iatrogenic sclerotomy retinal tears. Retina. 1997; 17(5):430-3.

13. Yepremyan M, Kapusta MA. The rate of iatrogenic retinal breaks in 25-gauge system pars plana vitrectomy. Invest Ophthalmol Vis Sci. 2004;45: E-Abstract 1951.

14. Lam DSC, Yuen CYF, Tam BSM, Cheung BTO, Chan WM. Sutureless vitrectomy surgery [letter]. Ophthalmology. 2003;110(12):2428.

15. Desai UR, Alhalel AA, Schiffman RM, Campen TJ, Sundar G, Muhich A. Intraocular pressure elevation after simple pars plana vitrectomy. Ophthalmology. 1997;104(5):781-6.

16. Okamoto F, Sakata N, Hiratsuka K, Okamoto C, Yamane N, Oshika T. Changes in corneal topography following 25-gauge transconjunctival sutureless vitrectomy and 20-gauge standard vitrectomy. Invest Ophthalmol Vis Sci. 2004;45: E-Abstract 2862.

17. Fujii GY, de Juan Jr E, Humayun MS, Chang TS, Pieramici DJ, Barnes A, et al. Initial experience using the transconjunctival sutureless vitrectomy system for vitreoretinal surgery. Ophthalmology. 2002;109(10):1814-20.

18. Virata SR, Kylstra JA. Postoperative complications following vitrectomy for proliferative diabetic retinopathy with sew-on and noncontact wide-angle viewing lenses. Ophthalmic Surg Lasers. 2001;32(3):193-7. 